Supporting Information

\title{
Metallic nanomesh with disordered dual-size apertures as wide viewing angle transparent conductive electrode
}

Tengfei Qiu', Bin Luo ${ }^{\ddagger}$, Fawad Ali ${ }^{\dagger}$, Esa Jaatinen ${ }^{\dagger}$, Lianzhou Wang ${ }^{\ddagger}$, Hongxia Wang ${ }^{* \dagger}$

†School of Chemistry, Physics and Mechanical Engineering, Queensland University of Technology, Brisbane, QLD 4001, Australia

${ }^{\ddagger}$ Nanomaterials Centre, School of Chemical Engineering and Australian Institute for Bioengineering and Nanotechnology, The University of Queensland, Brisbane, QLD, 4072, Australia

*corresponding author email address: $\underline{\text { hx.wang@qut.edu.au }}$ 


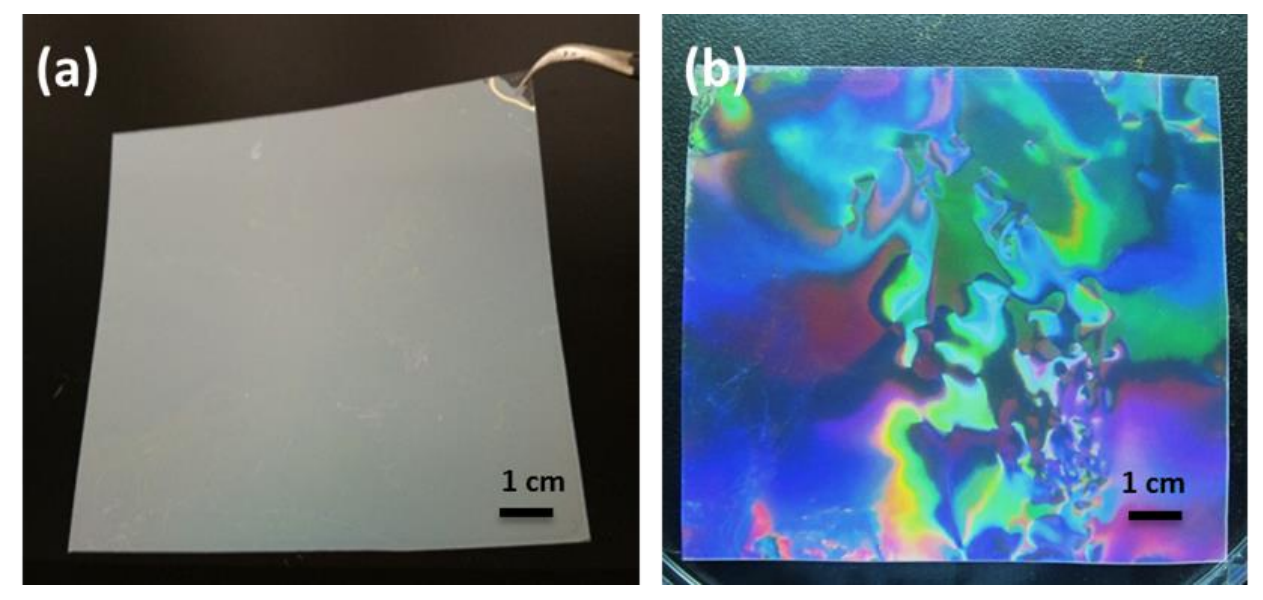

Figure S1. Photographs of a monolayer of (a) disordered dual-size (750 nm and $500 \mathrm{~nm})$ and (b) ordered single-size $(750 \mathrm{~nm})$ PS particles on PET substrates.

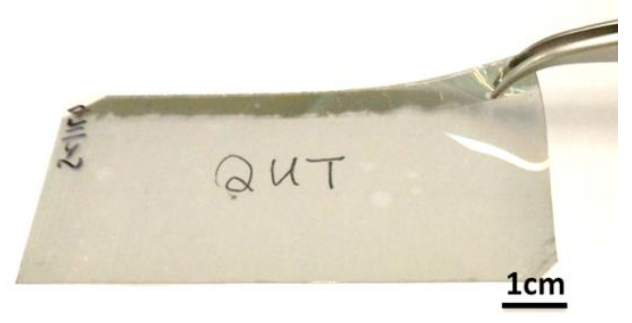

Figure S2. Photograph of Au nanomesh with dual-size apertures on a polyethylene terephthalate (PET) substrate.
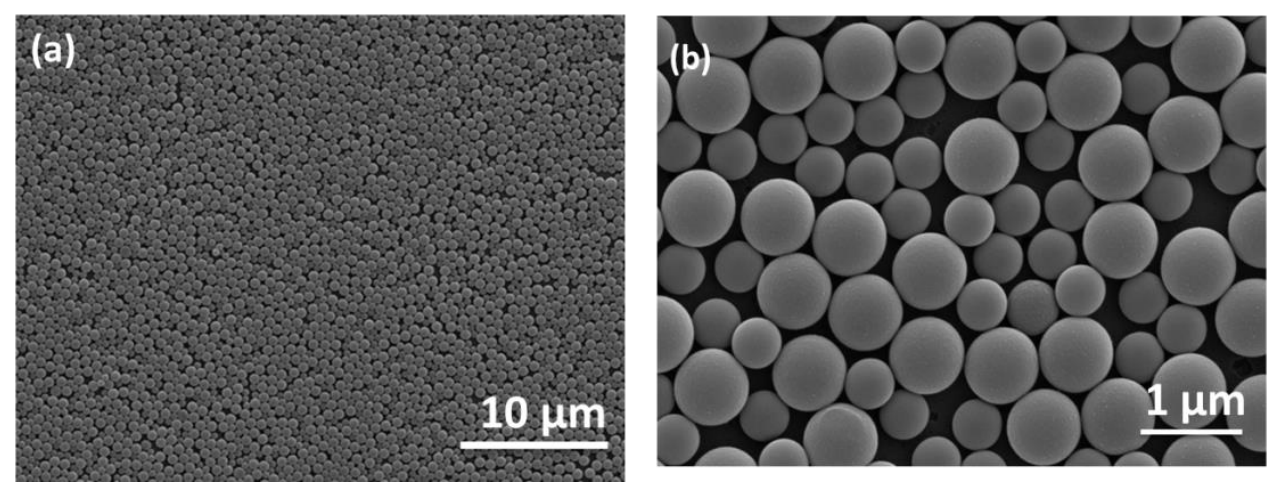

Figure S3. SEM micrographs of the monolayer of dual-size PS particles at (a) low- and (b) highmagnifications. 


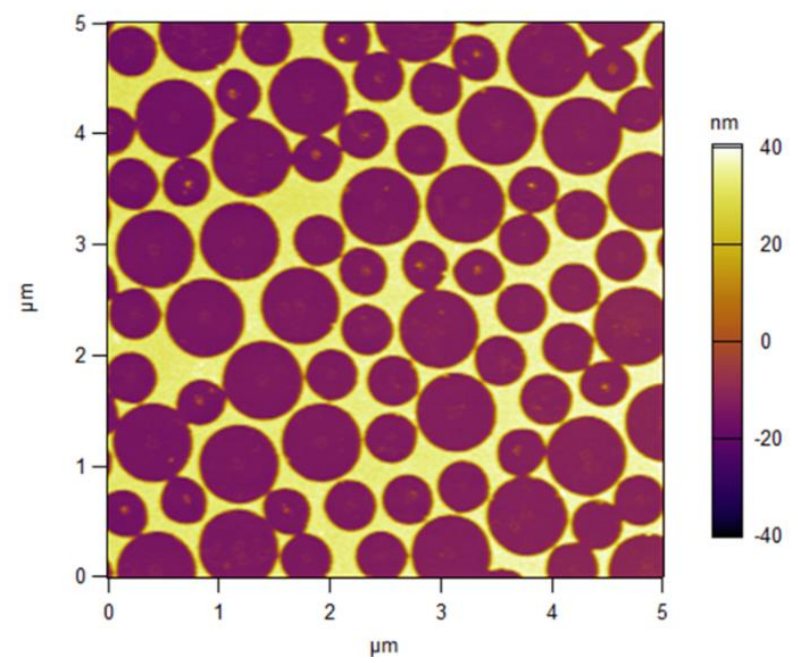

Figure S4. AFM image of a $50 \mathrm{~nm}$ Au film with disordered dual-size apertures on a glass substrate.

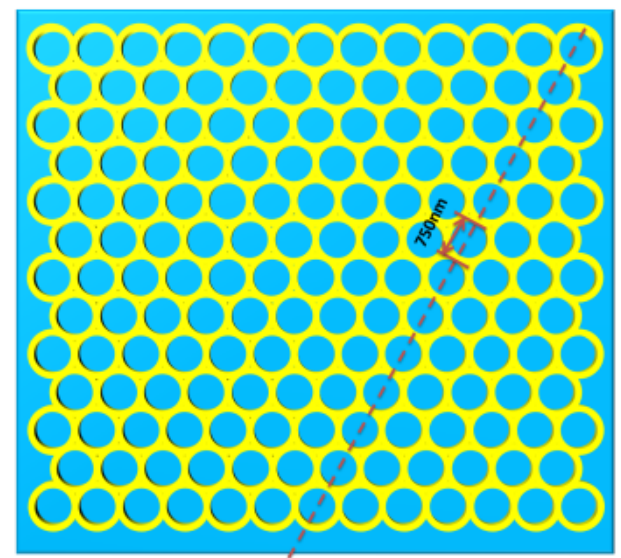

Figure S5. Schematic diagram of the metallic nanomesh with ordered single-size apertures. The red dash line shows the direction where the apertures are closely aligned. The period of the pattern along this direction is $750 \mathrm{~nm}$.

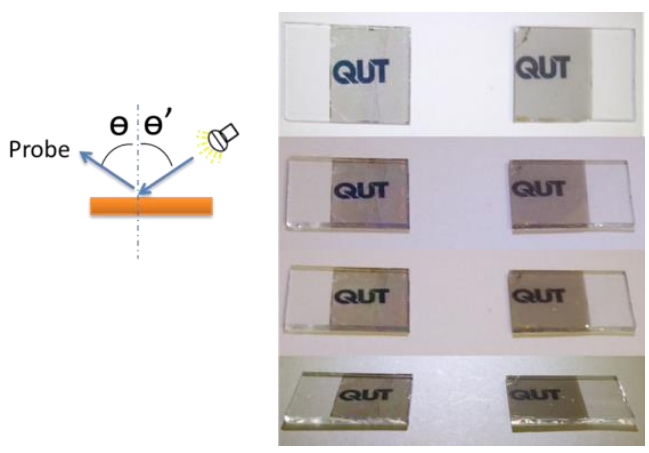

Figure S6. Optical images showing the structural color of the Au nanomesh composed of ordered single apertures (left) and composed of disordered dual-size apertures (right) when the light incidence angle equals the viewing angle. 
Table S1. Parameters of the diffraction grating equation $(\mathrm{m}=1)$

\begin{tabular}{llll}
\hline$d(\mathrm{~nm})$ & $\lambda(\mathrm{nm})$ & color & $\theta\left(^{\circ}\right)$ \\
\hline 750 & $390-455$ & violet & $31.3-37.3$ \\
750 & $455-492$ & blue & $37.5-50$ \\
750 & $492-577$ & green & $50-50.3$ \\
750 & $577-597$ & yellow & $50.3-52.8$ \\
750 & $597-622$ & orange & $52.8-56$ \\
750 & $750-622$ & red & $56-90$ \\
\hline
\end{tabular}
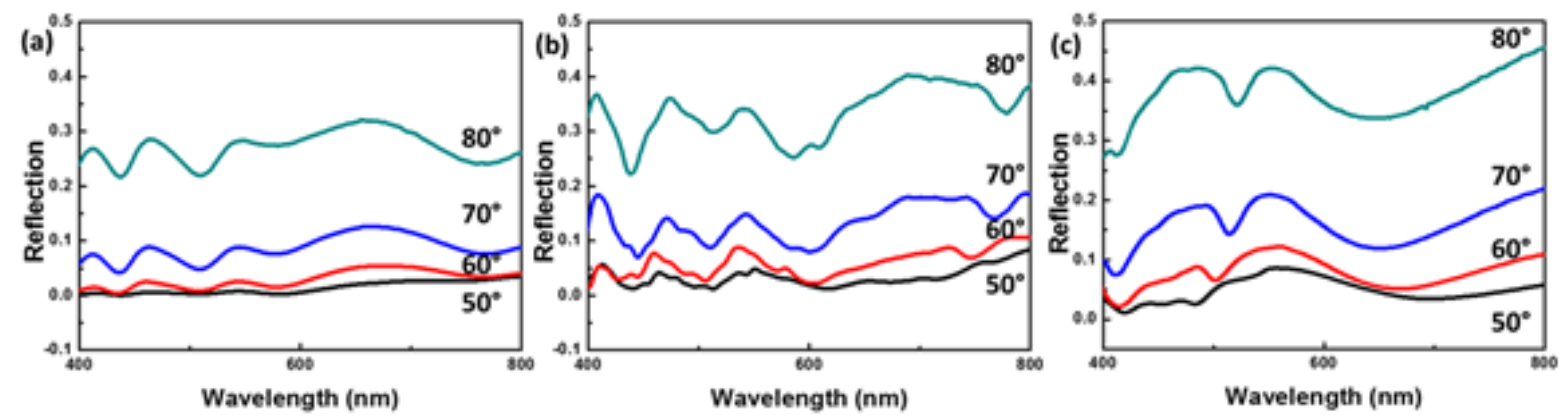

Figure S7 Reflection spectra of the monolayer of (a) $750 \mathrm{~nm}$ and $500 \mathrm{~nm}$ mixture, (b) $750 \mathrm{~nm}$ and (c) 500 nm PS spheres on glass substrates.

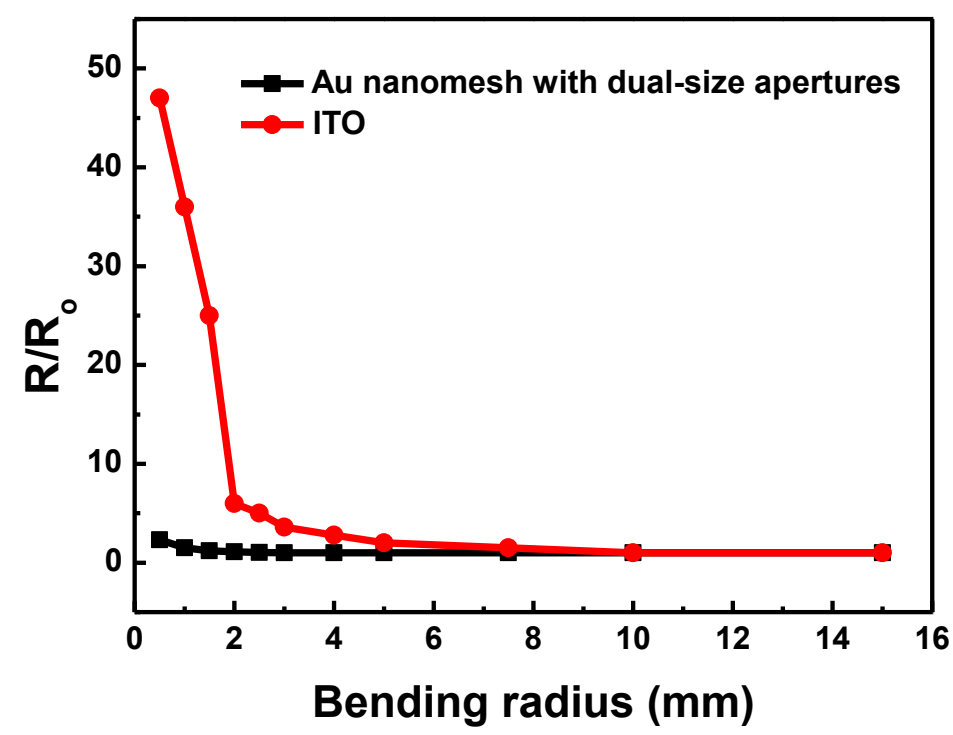

Figure S8 The resistance change of the Au nanomesh film with dual-size apertures ( $R s=55 \Omega s^{-1}, T=72 \%$ ) and the ITO film on PET substrates after one time bending and recovering at different bending radius. 